\title{
American Pediatric Society Presidential Address 2007: Robust Complex Networks in Health, Disease and International Pediatric Research
}

\author{
EDWARD R. B. MCCABE \\ Mattel Children's Hospital, The University of California - Los Angeles, Los Angeles, CA 90095, Departments of Pediatrics and Human \\ Genetics, David Geffen School of Medicine; Department of Bioengineering, Henry Samueli School of Engineering and Applied Science, \\ The University of California - Los Angeles, Los Angeles, CA 90095; UCLA Center for Society and Genetics, and California NanoSystems \\ Institute (CNSI), The University of California - Los Angeles, Los Angeles, CA 90095
}

I am honored to have served you as the president of the American Pediatric Society (APS) in its $119^{\text {th }}$ year, and I will always appreciate in so many ways your willingness to place your trust in me.

I feel that we are at a critical juncture in the evolution of the APS as a force to encourage international development of pediatric research beyond the current confines of the International Pediatric Research Foundation (IPRF), specifically North America and Europe. To that end, at the Fall APS Council meeting I established the Japan - Pacific Rim Working Group, which will report its recommendations to the APS Council and the Joint Council meetings no later than the Fall 2009 meetings. In this address, I will provide to you my rationale for expanding the reach of organized pediatric research internationally, and why it is essential for the APS to play an important part in this expansion. Fundamental to the understanding of this expansion and its importance is an appreciation for the organization and robust properties of scale-free networks.

\section{ROBUST, SCALE-FREE NETWORKS}

We began to study networks as a consequence of our research on glycerol kinase deficiency (GKD) and observations about genetic diseases more generally (1-3). We had cloned and characterized the $G K$ gene (4), and, in patients with $G K$ missense mutations, we had measured residual GK activities and mapped their mutations to a model of the GK protein (5). Patients with isolated GKD (meaning they have mutations within the $G K$ gene) are either symptomatic with episodes of metabolic and neurologic deterioration or "asymptomatic" with no clinical phenotype until some develop insulin resistance and type 2 diabetes mellitus in adulthood (6). We found no correlation between genotype and phenotype for patients with GKD (5) and concluded that this was due at least in part to the complexity of networked systems.

We determined that we must understand systems dynamics and the interactions of biologic networks if we are to under-

Received June 7, 2007; accepted June 12, 2007.

Correspondence: Edward RB McCabe, M.D., Ph.D., Mattel Children's Hospital at UCLA, 10833 LeConte Ave, 22-412 MDCC, Los Angeles, CA 90095-1752, Phone: (310) 825-5095, Fax: (310) 206-4584; Email: emccabe@ mednet.ucla.edu

Presented at the 2007 Annual Meeting of the Pediatric Academic Societies, Toronto, Ontario, Canada. stand the pathogenesis of GKD and other genetic diseases (3). We also learned that these networks are important beyond fundamental biology $(7,8)$.

There are two alternative possibilities for networks regarding their sensitivity or tolerance to variataition (9). If a network is highly sensitive to internally or externally imposed changes, then the precise selection of all parameters would be required to maintain stability of the network. On the other hand, if a network tolerates change, then the robust properties of key components would be determined by systems dynamics within much broader ranges of parameters.

The structures of networks can be classified as either random or scale-free $(10,11)$. Random networks are relatively homogeneous with respect to the connectivity of the hubs, meaning that most nodes have a similar number of connections, i.e., the numbers of connections for the hubs represents a normal distribution over a relatively narrow range. Scale-free networks are more hierarchical in their connectivity with most of the hubs having a low number of connections and only a small number of hubs having a high number of connections. Scale-free networks are also known as "hub and spoke" networks, and this architecture makes them extremely robust with a high tolerance to changes in network traffic and random failures of nodes.

Biologic networks. Scale-free, hub and spoke networks are observed in biology (3,9-14), and individual network modules are linked together to form the modular structures of cells, tissues and organisms $(10,12)$. The architectural features of these networks that provide their robust tolerance to error and attack, also lead to their vulnerability (11). Attacks on highly connected nodes fragment the network and decrease its ability to survive, and knowledge of this vulnerability allows us to understand disease pathogenesis and therapeutic intervention $(3,11)$. Networked biologic modules will have properties analogous to those in engineering and computer science, such as amplifiers and logic circuits, and identification of general principles underlying these scale-free networks will permit functional models to be understood and manipulated (12).

We began to recognize that the phenotypes of "simple," "single" gene disorders are complex traits due at least in part to the roles of genetic and environmental modifiers on the systems dynamics of these robust scale-free networks (1-3). When mutations are considered within the networks in which 
these proteins normally operate, if a highly connected hub is impacted then the result is the elimination of an entire segment of the network (13).

Consideration of networked biology has led us to investigations in systems biology $(14,15)$. Research by our group and others is moving biology from a qualitative to a mathematical discipline and involves the examination of the totality of this networked biology, not just one gene or protein at a time. These investigations require identification of network components and quantification of network activity.

Human-designed networks. Networks that are constructed by humans, and have a robust tolerance to nodal failures and network volumes are organized with hub and spoke architectures (7). For example, the internet and world-wide web have this structure, and both show vulnerability to attack on highly connected hubs consistent with them being scale-free networks (11) (e.g., www.nd.edu/ networks/Image\%20Gallery/ gallery.htm). Many airlines are organized in this manner, with the hub airports fed by the parent and regional carriers (www. airlineroutemaps.com/). Accordingly, weather can interrupt traffic at the peripheral, low connectivity nodes without significant effect on the network, as long as the major, highly connected hubs remain active. Similarly, the land connectivity of population centers as seen at night from space appears to form a scale-free network with well-lighted hubs connected by lighted spokes (www.nasa.gov/vision/earth/everydaylife/Growing_Cities_ AGU_prt.htm).

Sustainable social networks. For social networks to be sustainable they must be robust and tolerant to change, including times when one whole segment of a network may not be functioning, perhaps because of loss of communication with that segment. These are the properties of scale-free, hub and spoke networks, and therefore sustainable social networks will have the same design properties we have seen in biologic and human-designed scale-free networks.

\section{International Pediatric Research Network}

Why am I discussing networks at the meeting of the Pediatric Academic Societies (PAS)? I would argue that we need sustainable social networks in the international pediatric research community, and expanding these networks has been the focus of my APS presidency. To that end I have established the Japan-Pacific Rim Working Group (JPRWG). The JPRWG will make a recommendation regarding the expansion of this international network to the APS Council and then to the APS-Society for Pediatric Research (SPR) Joint Council meeting no later than the Fall Council Meetings in 2009. The success of the PAS meeting in Honolulu, Hawa'i (May 3-6, 2008), particularly in attracting attendees from Asia, will be critical to our recommendation.

Purpose. The purpose of an expanded international pediatric research network will be multi-fold. It will be more representative internationally, and will increase the speed and breadth of distribution for research results internationally. This expansion will also make the pediatric research community more robust. Most importantly, it will improve the care of children internationally by accelerating the translation of research into clinical practice.
I would argue that we should look toward Asia for this expansion because that is where the largest populations are currently located and will be increasing most rapidly with the addition of even more children to these populations. Among the 10 countries with the highest populations, all greater than 100 million people, six are in Asia: China, India, Indonesia, Pakistan, Japan and Bangladesh (Table 1) (16). According to United Nations estimates, despite declining fertility rates, at all but the lowest estimates of fertility, the world's population will continue to grow through 2050, and the region with the largest population growth will be Asia (17). The top three countries in population growth are in Asia: India, China and Pakistan (Table 2) (17).

$\boldsymbol{H u b}$ nodes. If we look at the organization of pediatric research societies internationally, then we can see hub and spoke network structures emerge. For example, the International Pediatric Research Foundation (IPRF) is a hub node, with the additional nodes in the network consisting of two North American organizations, the SPR with its regional satellites and the APS, and one European organization, the European Society for Pediatric Research (ESPR) (Fig. 1A). As the only major organization with the words "international," "pediatric" and "research" in its name, it is interesting that the IPRF member societies are limited to North America and Europe. The PAS organizes and convenes annual meetings and therefore, serves as a hub node for the four PAS Sponsors and 12 PAS Alliance Organizations, including the Asian Society for Pediatric Research (ASPR) and the Japan Pediatric Society, which was founded in 1896 and is the second oldest pediatric organization in the world after the APS (Fig. 1B).

Proposal. We have reviewed the data that show that six of the ten counties with the highest current populations are in Asia and the greatest population growth will be in Asia. Therefore, the largest numbers of children are and will be in Asia, but the pediatric research community of Asia is currently not included in the IPRF.

I propose immediate expansion of the IPRF membership to include the 111-y-old JPS (Fig. 1C). In addition, membership for the ASPR should be considered by the IPRF (Fig. 1C). The ASPR is a young organization that held its second annual meeting in Yokohama, Japan, in December 2006, and when I attended the ASPR Council meeting in Yokohama I was

Table 1. Countries with highest current populations, all greater than 100 million

\begin{tabular}{ll} 
China & Pakistan \\
India & Russia \\
USA & Japan \\
Indonesia & Bangladesh \\
Brazil & Nigeria \\
\hline
\end{tabular}

Those in bold are in Asia.

Table 2. Top three countries in population growth

\begin{tabular}{lcc}
\hline Country & $1995-2025$ & $1950-2050$ \\
\hline 1. India & 401 & 1,180 \\
2. China & 260 & 962 \\
3. Pakistan & 133 & 318 \\
\hline
\end{tabular}



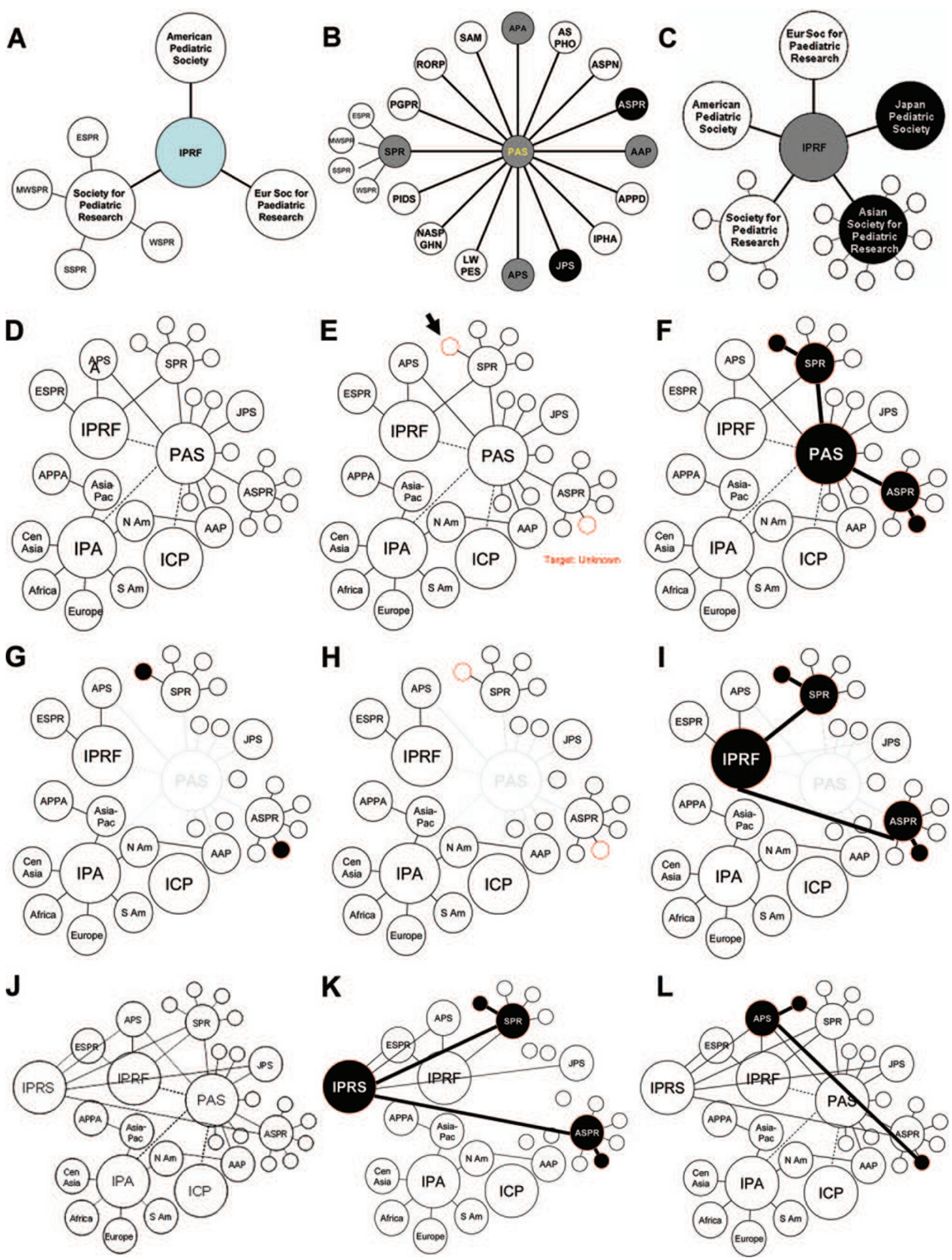

Figure 1. Row 1 - Existing and proposed organization of pediatric research societies internationally. A. The International Pediatric Research Foundation (IPRF) is a hub node with peripheral nodes, specifically the SPR and its regional societies, the APS and the ESPR. The regional societies of the SPR include the Eastern SPR (ESPR), the Midwest SPR (MWSPR), the Southern SPR (SSPR) and Western SPR (WSPR). B. The PAS is a convener for an annual meeting bringing together the four PAS sponsors and 12 PAS Alliance Organizations, including the Asian Society for Pediatric Research (ASPR) and the Japan Pediatric Society 
impressed by their plan to establish a secure future. ASPR membership would provide a broader reach into Asia for the IPRF. In addition, the ASPR may want to consider developing regional satellites analogous to the SPR regional societies for pediatric researchers who may not have the financial ability to travel to the annual ASPR meetings.

A relatively robust network of international organized pediatrics is currently in existence that includes the pediatric research societies, particularly if one considers the fact that meetings of individuals associated with the IPRF, the International Pediatric Association (IPA) and the International Congress of Pediatrics (ICP) often occur at the PAS meeting (Fig. 1D). A social network, such as the international pediatric network, can be searched even when the identity of the target may not be known to the individual who initiates the search $(7,8)$. For example, an investigator in one of the regional societies associated with the SPR may have an interest in the details of an unpublished protocol for research on a disease found in a particular area of Asia (Fig. 1E). By probing contacts at the SPR, who direct the initiating investigator to individuals for discussions at the annual PAS meeting, followed by queries to the membership of the ASPR, the target in a regional affiliate of the ASPR could be identified (Fig. $1 F)$. This hypothetical search involved four spokes, or, in the mathematical terminology of networks, "edges," and these four edges represent the distance from initiator to the target.

The vulnerability of a network to the loss of a critical node is measured by the diameter of the network or the number of edges required to traverse the network, and, if the network diameter (the distance across the network) increases substantially, then the compromised network is described as fragmented (11). In the example of the international pediatric network we could model the cancellation of the annual PAS meeting, perhaps if the meeting was planned in a city paralyzed by a natural disaster, such as an earthquake, immediately before the meeting (Fig. 1G). In this model, based on the existing international pediatric network (Fig. 1D), in which all of the connections between the PAS and other societies are lost, there would be no opportunity for the random enlightening discussions at the annual meeting, and there is no pathway connecting the North American investigator with the unknown investigator in Asia (Fig. 1G). The diameter of the international pediatric network would increase to infinity and the network would be completely fragmented. Expansion of the IPRF to include the JPS and ASPR (Fig. 1C) would provide an alternative to the PAS with the IPRF serving as another central, highly connected node (Fig. 1H). This expanded IPRF would generate an even more robust architecture for the international pediatric research community. For example, in the modeled cancellation of an annual PAS meeting (Fig. 1G), the IPRF expansion would connect the North American initiating investigator to the unknown target investigator in Asia with an identical number of four edges (Fig. 1I) as the path through the PAS (Fig. 1F) - the diameter of the network would remain the same and the network would no longer be fragmented by cancellation of the PAS meeting. Therefore, the expanded IPRF would make the entire pediatric community more robust than it is in its current configuration.

The pediatric research community and the children we serve need a more robust international network, which could be provided by the proposed expansion of the IPRF, or, if the IPRF chooses not to expand, then through an alternative organizational structure, referred to here as the International Pediatric Research Society (IPRS) (Fig. 1J). This would provide a path from the inquiring North American to the target

Figure 1 (Continued). (JPS) (shown in black). C. Proposed expansion of the International Pediatric Research Foundation (IPRF). This proposal should include the JPS and the ASPR (shown in black) to extend the reach of the IPRF beyond North America and Europe.

Row 2 - Existing relatively robust international pediatric network. D. A relatively robust network of international pediatric organizations currently exists that includes the pediatric research societies. The network connectivity is increased by meetings of individuals associated with the International Congress of Pediatrics (ICP), International Pediatric Association (IPA) and IPRF that may occur at the annual PAS meeting (shown by dashed lines). The IPA is composed of numerous national, regional/linguistic and subspecialty societies that I have indicated here by region. These regions are represented by official liaison organizations, such as the American Academy of Pediatrics (AAP) representing US pediatric societies in the North American (NAM) region and the Asian Pacific Pediatric Association (APPA) for the Asian Pacific region. Other abbreviations include Cen Asia for Central Asia and S Am for South America. E. One of the characteristics of social networks is that they can be searched even when the originator of the search (in this example a member of a regional society of the SPR, shown by the arrow) with an interest in a disease in Asia, even when the target (in this example a member of a proposed regional society of the ASPR) is unknown $(7,8)$. F. The path of the search involves four links (referred to in the mathematical language of networks as "edges") connecting the search originator's organization to the SPR, PAS, ASPR and the target investigator's organization. Theses four edges are a measure of the length of this search path.

Row 3 - Proposed even more robust international pediatric network via expansion of the IPRF. G. The robustness of a scale-free network is determined by the consequences of the loss of a highly connected node, in this example through a disruption of an annual PAS meeting (indicated in gray). With the existing network (based on Fig. 1D), there would be no path to connect the investigators in regional North American and Asian societies (shown in black). H. The proposed expansion of the IPRF, with addition of the JPS and ASPR would provide an alternative highly connected central pediatric research node. I. The expanded IPRF would provide a pathway from the North American to the Asian investigator with the same four edges as the path through the PAS (as determined in Fig. 1F).

Row 4 - Establishment of alternative organizational structure if the IPRF chooses not to expand. J. A more robust international pediatric research network is required, and if the IPRF chooses not to expand into Asia, then another organization, such as the International Pediatric Research Society (IPRS) will be formed to fill this need. It is hoped that the current member organizations of the IPRF would join this new society, as shown here. K. Assuming that the SPR would be a member organization of the IPRS, then the IPRS would provide an alternative route between the North American and Asian investigator with the same path length of four edges as through the PAS (Fig. 1F) or IPRF (Fig. 1I). L. Small world phenomenon in international pediatric research. I will use the same search model described in Fig. 1E with an initiating investigator in North America and a target investigator in Asia. Perhaps a colleague at a regional society of the SPR knows of my travels to Asia and connects the initiating investigator to me through the APS. If I met the target at the 2006 ASPR meeting that I attended then I could connect the North American and Asian Investigators with only two edges. This small world phenomenon would represent a decrease in path length by $50 \%$ compared with paths through the PAS (Fig. 1F) or IPRF (Fig. 1I). 
Asian investigator with four edges and therefore no increase in network diameter (Fig. 1K).

Small world phenomena in international pediatric research. In 1967, Harvard University social psychologist Stanley Milgram developed the small-world method (8). Randomly selected individuals from Omaha, Nebraska, were given letters and the target was a stockbroker in Boston, Massachusetts. They were instructed to send the letters only to someone who was known to them on a first name business, and to someone they thought was closer to the target. The number of steps required to reach the target was estimated to be in the hundreds, but the actual number of steps was approximately six. This is the origin of the phrase, "six degrees of separation," the popularly recognized terminology that we use when we encounter an example of the small world phenomenon in our own experience.

The basis for small world phenomena is the difference between the way we perceive individuals' identities and the complexity of these identities (8). We generally consider one's identity to be determined by two attributes, commonly current occupation and geography. A small world experience occurs through another aspect of the individual's identity, such as a previous geographic location for school or work, an unanticipated social affiliation, a hobby or other attribute, outside of the accepted two-dimensional framework for identity. In effect, the routine two-dimensional process of social interconnectivity is "short-circuited" by attributes in additional social dimensions.

Let us consider how strengthening interactions within the international pediatric research community can improve the speed of communication through small world phenomena. We will use the same social probing model of the initiating investigator in North America and target investigator in Asia that we have used previously (Fig. 1E). The initiating investigator may ask colleagues at one of the SPR regional meetings if they have any ideas about how to identify the target (Fig. 1L). One of these colleagues may know of my numerous recent visits to Asia, including the second annual meeting of the ASPR. If I met an investigator there from the same region as the target investigator, then these personal relationships may lead to achieving the goal of this query with only two edges. This would mean that building personal international interactions with our colleagues in Asia will accelerate communication through small world phenomena, in this example decreasing the network diameter by $50 \%$ from four to two edges, doubling the speed of communication.

Expanding our interpersonal networks internationally will increase the opportunities for small world moments, improving the connectivity among researchers in pediatrics and strengthening pediatric research worldwide. This will accelerate the translation of research into improved health care for children internationally. In addition, at the personal level, it is enjoyable and rewarding to get to know new colleagues and new cultures as we build these professional relationships.

The value of social networking. Robert Metcalfe, the inventor of the Ethernet, formulated the eponymic relationship regarding telecommunications networks (18). The Metcalfe law maintains that a network's value increases in proportion to the square of the number $\left(\mathrm{n}^{2}\right)$ of users for a telecommunications network or members for a social network. This law establishes the basis for online social networking communities such as MySpace and Facebook.

According to the Metcalfe law, therefore, an expanded international pediatric research network will have increased value for its members, pediatric investigators. But I would assert that the researchers are proxies for the individuals who will benefit most from the amplified power of the network expansion, the children.

Splitting a larger network or maintaining a fragmented network has been considered a reversal of the Metcalf law (http://www.useit.com/alertbox/990725.html). If a potentially larger network remains partitioned into $\mathrm{N}$ isolated fragments, then each of these components will have a size of $1 / \mathrm{N}$ and a value $1 / \mathrm{N}^{2}$ compared with the consolidated, more robust network. Therefore, maintaining the international pediatric research network in its current configuration, which does not include the growing populations of children in Asia, is excluding the value of this larger network from the world.

\section{FUTURE OPPORTUNITIES AND MESSAGES FOR THE APS}

We have the opportunity to work together to build a more robust, higher value, international pediatric research network. For the sake of children all over the world, including those in North America and Europe, as well as those who will be embraced by the proposed and subsequent network expansions, we are obligated to make every attempt to achieve this opportunity.

Dr. John Howland, considered "the leading pediatrician of this country, if not in the world" (after whom the highest award of the APS is named), demonstrated that progress in pediatric clinical care could be accelerated by integrating basic science methodologies into pediatric research (19). We, the members of the APS, must continue Howland's legacy of improving pediatric care through research innovation by looking beyond North America and Europe to integrate pediatric research in a robust international network.

I will close with several messages for the APS based on consideration of robust, scale-free networks. The APS must be a well-connected hub, and not a peripheral expendable node. The APS will be stronger and more valuable as part of a larger, integrated system, and we should resist any forces that would encourage our insularity. Therefore, we must all work to increase the value and power of the pediatric research network by strategic expansion worldwide. Along the way, we need to mentor and be mentored by our international relationships. And be sure to enjoy the "small world surprises!"

Thank you again for placing your trust in me and honoring me as your president.

\section{REFERENCES}

1. Dipple KM, McCabe ER 2000 Phenotypes of patients with "simple" Mendelian disorders are complex traits: Thresholds, modifiers and systems dynamics. Am J Hum Genet 66:1729-1735

2. Dipple KM, McCabe ER 2000 Modifier genes convert "simple" Mendelian disorders to complex traits. Mol Genet Metab 71:43-50 
3. Dipple KM, Phelan J, McCabe ER 2001 Consequences of complexity within biological networks: Robustness and health, vulnerability and disease. Mol Genet Metab 74:45-50

4. Guo W, Worley K, Adams V, Mason J, Sylvester-Jackson D, Zhang Y-H, Towbin JA, Fogt DD, Madu S, Wheeler DA, McCabe ER 1993 Genomic scanning for expressed sequences in Xp21 identifies the glycerol kinase gene. Nat Genet 4:367-372

5. Dipple KM, Zhang Y-H, Huang B-L, McCabe LL, Dallongeville J, Inokuchi T, Kimura M, Marx JH, Roederer GO, Shih V, Yamaguchi S, Yoshida I, McCabe ER 2001 Glycerol kinase deficiency: Evidence for complexity in a single gene disorder. Hum Genet 109:55-62

6. McCabe ER 2001 Disorders of glycerol metabolism. In: Scriver CR, Beaudet AL, Sly WS, Valle D (eds) The Metabolic and Molecular Basis of Inherited Disease (8th Edition). McGraw-Hill, New York, pp 2217-2237

7. Buchanan M 2002 Nexus: Small Worlds and the Groundbreaking Theory of Networks. W.W. Norton and Company, New York

8. Watts DJ 2003 Six Degrees: The Science of a Connected Age. W.W. Norton and Company, New York

9. Barkai N, Leibler S 1997 Robustness in simple biochemical networks. Nature 387:913-917

10. Barabasi AL, Oltvai ZN 2004 Network Biology: understanding the cell's functional organization. Nat Rev Genet 5:101-113
11. Albert R, Jeong H, Barabasi AL 2000 Error and attack tolerance of complex networks. Nature 406:378-382

12. Hartwell LH, Hopfield JJ, Leibler S, Murray AW 1999 From molecular to modular cell biology. Nature 402:C47-C52

13. Clipsham RC, McCabe ER 2001 Single tube gene-specific expression analysis by higher primer density multiplex reverse transcription. Mol Genet Metab 74:435-448

14. MacLennan NK, Rahib L, Shin C, Fang Z, Horvath S, Dean J, Liao JC, McCabe ER, Dipple KM 2006 Targeted disruption of glycerol kinase gene in mice: Expression analysis in liver shows alterations in network modules related to glycerol kinase activity. Hum Mol Genet 15:405-415

15. MacLennan NK, Dong J, Horvath S, McCabe ERB 2007 Network analysis of glycerol kinase deficient mice predicts genes essential for survival: A systems biology approach. Pediatric Academic Society abstract \#5715.2

16. Diamond J 2006 Collapse. Penguin Books, New York, p 511

17. Heilig GH 1996 World population prospects: analyzing the 1996 UN population projections. International Institute for Applied Systems Analysis (IIASA), LUC Project, WP-96-146 http://www.iiasa.ac.at/Research/LUC/Papers/gkh1/chap1.htm (Accessed June 7, 2007)

18. Malone MS 2007 Hooked up. UCLA Magazine http://www.magazine.ucla.edu/ features/25-brilliant-ideas/index21.html (Accessed June 7, 2007)

19. Davison WC 1950 John Howland: the seventy-fifth anniversary of his birth. J Hist Med Allied Sci 5:197-205

\section{Erratum}

In the article, "Lipid peroxidation, caspase-3 immunoreactivity, and pyknosis in late-gestation fetal sheep brain after umbilical cord occlusion" by Margie Castillo-Melendez et al. (Pediatr Res 55:864-871) the authors report that the values for the immunohistochemistry quantification are off by a factor of ten due to a calculation error. The statistics on the data are unchanged. The authors regret the error. 